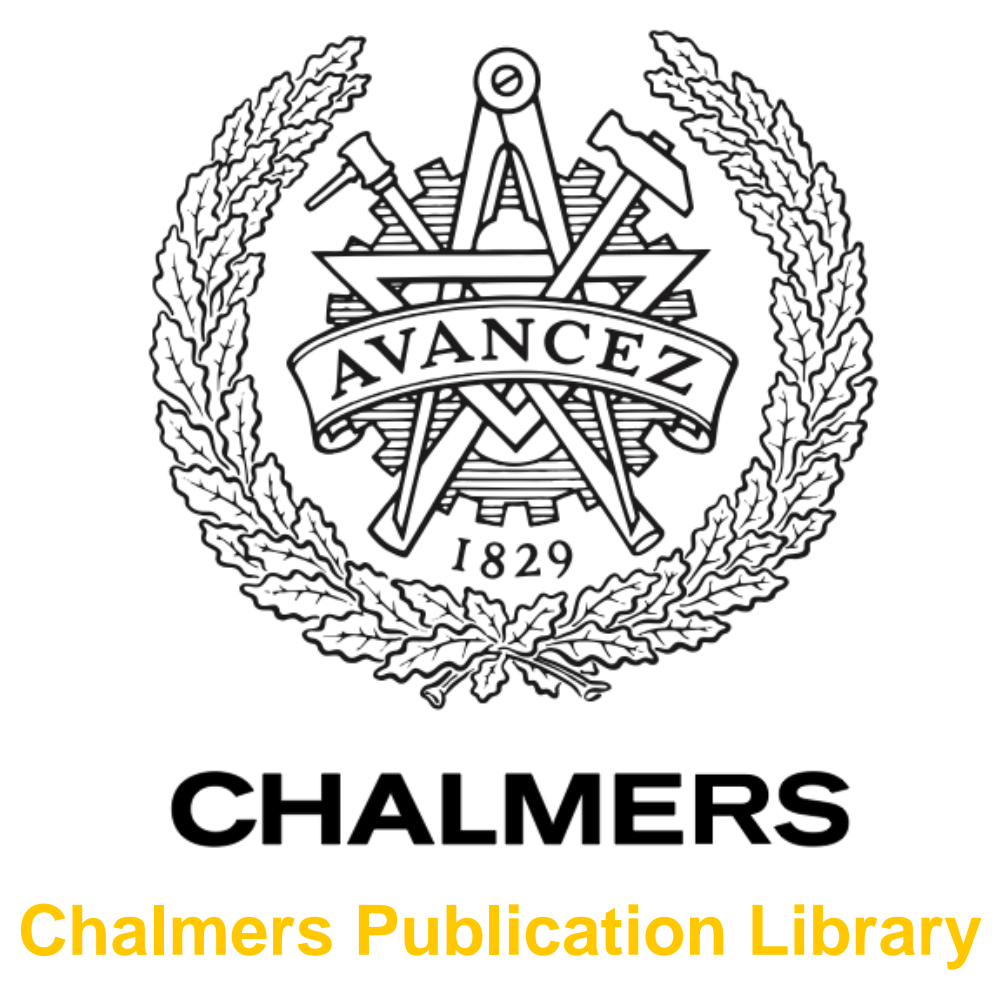

\author{
Novel ICI suppressing receiver for high-mobility DVB-T2 reception with large FFT \\ modes
}

This document has been downloaded from Chalmers Publication Library (CPL). It is the author's version of a work that was accepted for publication in:

Proc. IEEE International Symposium on Broadband Multimedia Systems and Broadcasting

Citation for the published paper:

Ochandiano, P. ; Wymeersch, H. ; Sobron, I. (2011) "Novel ICI suppressing receiver for high-mobility DVB-T2 reception with large FFT modes". Proc. IEEE International

Symposium on Broadband Multimedia Systems and Broadcasting

Downloaded from: http://publications.lib.chalmers.se/publication/151951

Notice: Changes introduced as a result of publishing processes such as copy-editing and formatting may not be reflected in this document. For a definitive version of this work, please refer to the published source. Please note that access to the published version might require a subscription.

Chalmers Publication Library (CPL) offers the possibility of retrieving research publications produced at Chalmers University of Technology. It covers all types of publications: articles, dissertations, licentiate theses, masters theses, conference papers, reports etc. Since 2006 it is the official tool for Chalmers official publication statistics. To ensure that Chalmers research results are disseminated as widely as possible, an Open Access Policy has been adopted.

The CPL service is administrated and maintained by Chalmers Library. 


\title{
Novel ICI Suppressing Receiver for High-Mobility DVB-T2 Reception with Large FFT Modes
}

\author{
Pello Ochandiano (pochandiano@eps.mondragon.edu), Henk Wymeersch (henkw@chalmers.se), \\ Iker Sobrón (isobron@eps.mondragon.edu), Lorena Martínez (lmartinez@eps.mondragon.edu), \\ Mikel Mendicute (mmendikute@eps.mondragon.edu)
}

\begin{abstract}
This paper proposes a novel framework for reducing the inter-carrier interference (ICI) caused by timevarying channels in mobile OFDM systems. The proposed ICI suppressing scheme is based on the application of the sumproduct (SP) algorithm to a factor graph (FG) representing the joint a posteriori probability (APP) of the transmitted symbols. Simulation results show that this new iterative soft-input soft-output (SISO) detection strategy achieves good performance when the ICI power is high, which can be due to high mobility or the use of large FFT sizes. We discuss its application to the reception of the new DVB-T2 broadcasting standard for a set of realistic mobile scenarios.
\end{abstract}

Keywords-OFDM, Time-varying channels, ICI suppressing, factor graph.

\section{INTRODUCTION}

DVB-T2 [1] is the world's most advanced digital terrestrial transmission system offering high efficiency, robustness and flexibility. This new specification has noteworthy increased robustness and spectral efficiency compared to its predecessor (DVB-T). The DVB-T2 specification makes use of low density parity-check (LDPC) codes to protect against high noise levels and interference. Regarding the modulation, the new standard uses the same orthogonal frequency division multiplexing (OFDM) system. Three cascaded forms of interleaving have been included among many novel signal processing stages. Furthermore, the DVB consortium has issued a Call for Technologies for the Next Generation Handheld (NGH) standard design with the aim of providing an advanced mobile broadcast system.

OFDM is a popular multi-carrier modulation method in which data is divided into several parallel streams or channels, one for each orthogonal subcarrier. Since each subcarrier is modulated at a low symbol rate, the insertion of a guard interval (GI) with a cyclic prefix provides the ability to equalize each subcarrier independently, coping with severe channel conditions without increasing the complexity of the equalizer. Some of the most recent wireless communications standards propose very large OFDM symbol lengths (e.g., 32K subcarriers in DVB-T2), which implies increasing the GI length without losing spectral efficiency, with the aim of enhancing the performance of the transmission over frequencyselective channels and single frequency networks (SFN).

One of the main drawbacks of OFDM mobile communication systems is its sensitivity to Doppler frequency shifts, which destroy the orthogonality among subcarriers giving raise to inter-carrier interference (ICI) and deteriorating severely the bit error rate (BER) performance. The signal-to-interference ratio (SIR) depends on the Doppler frequency and the OFDM symbol length [2], therefore ICI can be devastating when large FFT sizes are used. This can be the case of the DVB-T2 and DVB-NGH reception.

The large amount of ICI compensation schemes proposed in the literature [3] [4] [5] [6] can be classified following different criteria. We can distinguish between linear and non-linear equalizers, optimal and sub-optimal detectors, etc. Considering how the mobile reception problem is tackled, ICI mitigation schemes can be split into two groups: Those who consider the ICI a source of frequency diversity and try to exploit it in order to enhance the BER performance [3], and those which interpret ICI as an interference and pretend to subtract it out from the signal [4].

ICI mitigation has been also considered for the first generation DVB standards [7] [8], and recently a novel approach has been proposed for DVB-T2 and DVB-NGH reception [9], where a pre-equalizer operating in sub-blocks of the received OFDM block was proposed in order to combat the timevariation of the channel caused by the Doppler frequency shift. The main drawback of this approach is that pilot carriers can not be used for channel estimation.

In this paper, we propose a novel approach based on factor graphs and the so-called belief propagation (BP) algorithm [10] to deal with ICI mitigation in OFDM reception with large FFT sizes. This new scheme, based on a fully parallel algorithm, achieves good performance in the low SIR scenarios, which makes it a good candidate for its implementation in second generation DVB receivers. Simulation results are provided for a bit-interleaved LDPC-coded OFDM system, showing the good performance of the proposed equalizer in realistic mobile DVB-T2 reception.

\section{SYSTEM DESCRIPTION}

We consider a basic OFDM communication system in which $M$-ary coded symbols $\mathbf{X}=\left[X_{0}, \ldots, X_{N-1}\right]^{T}$ are obtained by encoding a random sequence of bits and mapping them on a multilevel constellation. After the inverse Fourier transform (IFFT), the transmitted signal at symbol time $n$ is given by 


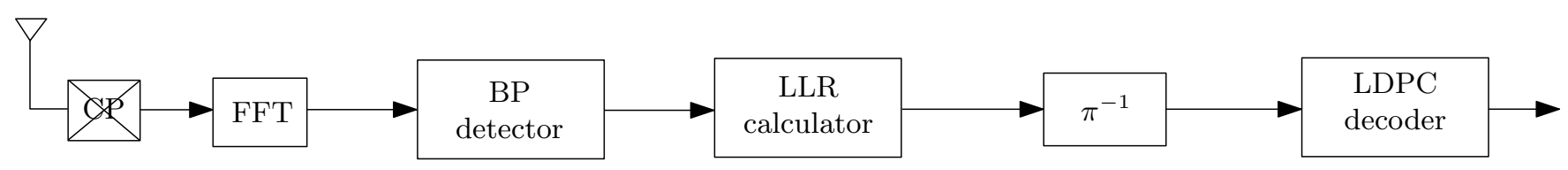

Fig. 1. Simplified Block diagram of a DVB-T2 reception chain including the belief propagation detector.

$$
x_{n}=\frac{1}{N} \sum_{k=0}^{N-1} X_{k} e^{j 2 \pi k n / N},
$$

where $N$ is the total number of subcarriers. Finally a cyclic prefix is added ensuring that no inter-symbol interference (ISI) is experienced.

The OFDM signal is transmitted over a doubly selective channel consisting of $L$ independent and identically distributed tap coefficients and a maximum Doppler frequency $F_{d}$. At the receiver, the signal $R_{k}$ at subcarrier $k$ after GI removal and fast Fourier transform (FFT) can be expressed as

$$
\begin{aligned}
R_{k} & =\sum_{n=0}^{N-1} r_{n} e^{-2 \pi n k / N} \\
& =\sum_{n=0}^{N-1} \sum_{l=0}^{L-1} h_{n, l} x_{n-l} e^{-j 2 \pi n k / N}+Z_{k},
\end{aligned}
$$

where $h_{n, l}$ is the $l$-th tap of the channel impulse response at time instant $n$, which is assumed perfectly known to the receiver. The additive white Gaussian noise (AWGN) sample $Z_{k}$ has complex variance $N_{0}$.

In matrix notation, the system model may be represented as

$$
\mathbf{R}=\mathbf{H X}+\mathbf{Z}
$$

where the $N \times N$ matrix $\mathbf{H}$ is the frequency response of the ICI channel. It has been proven that most of the energy of $\mathbf{H}$ is concentrated in the vicinity of the diagonal [2]. We define the ICI channel memory parameter $Q$ which determines the trade-off between performance and complexity of the detection algorithm. For many applications, $Q=3$ is considered (one off-diagonal value above and below the main diagonal of $\mathbf{H}$ ) for ICI suppressing.

\section{FACTOR GRAPH FRAMEWORK FOR ICI SUPPRESSING}

Based on the Bayesian estimation theory, the joint APP distribution of the transmitted symbols over an AWGN-affected random channel is expressed as

$$
P(\mathbf{X} \mid \mathbf{Y}) \propto P(\mathbf{Y} \mid \mathbf{X}) P(\mathbf{X}),
$$

where $P(\mathbf{Y} \mid \mathbf{X})$ represents the likelihood function and $P(\mathbf{X})$ is the a-priori distribution of $\mathbf{X}$. Factorizing the joint a-posteriori APP function into $N$ factors and assuming $Q=3$ permits to calculate the marginal $P\left(X_{k} \mid \mathbf{Y}\right)$ in a computationally attractive way as

$$
P(\mathbf{X} \mid \mathbf{Y}) \propto \prod_{k=1}^{N} f_{k}\left(X_{k-1}, X_{k}, X_{k+1}\right),
$$

where implicitly $X_{k}=0$ for $k \leq 0$ and $k \geq N$. Assuming Fourney's approach [11] for MAP sequence detection, each of the factors can be expressed as

$$
\begin{aligned}
f_{k}\left(X_{k-1}, X_{k}, X_{k+1}\right) & =P\left(R_{k} \mid X_{k-1}, X_{k}, X_{k+1}\right) \\
& \propto \exp \left\{-\frac{\left|R_{k}-\sum_{i=k-1}^{k+1} H_{k i} X_{i}\right|^{2}}{N_{0}}\right\} .
\end{aligned}
$$

In this paper, we further assume no a-priori information available. Fig. 2 depicts the detector's FG for $Q=3$, where each of the factor nodes are connected to three variable nodes. As it has been stated, the aim of the proposed algorithm is to compute marginal APPs for each transmitted symbol, which can be carried out applying the sum-product (SP) algorithm to the FG. The exchange of messages between factor and variable nodes representing the marginal APP mass functions of transmitted symbols exploits the frequency diversity introduced by ICI, enhancing the accuracy of exchanged messages. However, since the graph is not cycle free, the outputs of the algorithm are not exact marginal APPs.

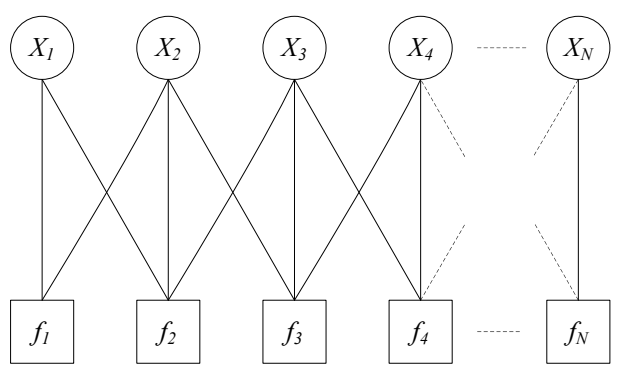

Fig. 2. Structure of the equalizer's factor graph corresponding to factor $f_{k}\left(X_{k-1}, X_{k}, X_{k+1}\right)$.

The effect of cycles in a FG has been extensively analyzed in the literature for many applications. It is well-known that the BER performance degrades severely when there are short cycles in the factor graph. Nevertheless, it has been shown that the average BER does not degrade significantly for some channel conditions, even with length 4 cycles [12]. From Fig. 2 , it is clear that the FG representing the ICI channel is girth 4 (the shortest loop is length 4). Nonetheless, simulation results will show that this is not a hindrance for a good BER performance. In this paper we quantify the performance 
degradation due to the presence of cycles in the graph by means of comparing the proposed algorithm with the optimal Bahl-Cocke-Jelinek-Raviv (BCJR) detector.

The FG-based framework has advantages over Trellis-based optimal detection schemes in terms of complexity and inherent structure suitability for large OFDM symbol detection. Because of its serial structure, BCJR algorithm would require a large amount of memory resources for storing the received signal while the detection process is being performed. Since we are interested in a fully parallel implementation, the socalled flooding schedule has been adopted: in each iteration, all factor and variable nodes transmit new messages to their neighbors.

\section{Complexity ANALysis}

The implementation of the log-domain SP algorithm can be carried out using the Jacobian logarithm, which only requires of summary operations and the evaluation of a nonlinear function by means of a lookup table. The complexity of the proposed algorithm is mainly a function of the following parameters: constellation size $(M)$, number of total subcarriers $(N)$ and ICI channel memory $(Q)$. Thus, the overall complexity scales as $\mathcal{O}\left(N Q M^{Q}\right)$.

Regarding the BCJR algorithm, the computation of $M^{\operatorname{div}(Q, 2)+1}$ values of the propagating message in the forward and backward recursions, requires summary operations involving $M^{Q}$ terms, whereas the computation of the marginal APPs requires a summary operation involving $M^{Q}$ terms.

\section{SIMULATION RESULTS}

In this section, simulation results show the performance of the proposed BP detector for several mobile scenarios in terms of BER versus SNR. The next table summarizes the main simulations parameters.

TABLE I

SIMULATION PARAMETERS

\begin{tabular}{cc}
\hline \hline Parameter & Value \\
\hline Modulation & QPSK \\
Number of subcarriers $(N)$ & 8196 \\
Subcarrier spacing & $1.12 \mathrm{KHz}$ \\
Length of one OFDM block $\left(T_{u}\right)$ & $896 \mu \mathrm{s}$ \\
Length of the guard interval $\left(T_{u} / 4\right)$ & $224 \mu \mathrm{s}$ \\
Channel coding & eIRA codes $(64800, \mathrm{R}=2 / 3)$ \\
\hline
\end{tabular}

Simulations have been carried out for two channel models: 6-taps Typical Urban (TU6) channel and 6-taps Rural Area. Three high-mobility scenarios have been considered where the normalized Doppler frequency $\left(F_{d} / \Delta f\right)$ is $0.15,0.3$ and 0.4 , where $\Delta f$ is the subcarrier spacing. The first one corresponds to about $200 \mathrm{~km} / \mathrm{h}$ of vehicular speed for the $8 \mathrm{~K}$ OFDM mode, and the last one represents about $160 \mathrm{~km} / \mathrm{h}$ of vehicular speed for the $32 \mathrm{~K}$ OFDM mode. Min-sum algorithm is used for LDPC decoding and perfect synchronization and ideal channel state information (CSI) are assumed at receiver.

First, in Fig. 3, we evaluate the aforementioned mobile scenarios and compare the BER performance against the free-ICI case for the TU6 channel. 10 iterations have been performed in the BP detector in order to assure the convergence of the algorithm, which means that carrying out more iterations does not improve the BER performance. We can appreciate that for $f_{d}=0.13$, the BP detector is very closed to the freeICI case, whereas the performance degrades significantly for higher Doppler frequencies. The reason for this behavior is that we consider the shortest ICI channel memory $(Q=3)$, consequently there is a residual ICI described by the rest of the elements in matrix $\mathbf{H}$, whose power increases with $f_{d}$. Increasing the channel memory would reduce the residual ICI and enhance the performance, although it would also increase the complexity of the algorithm exponentially.

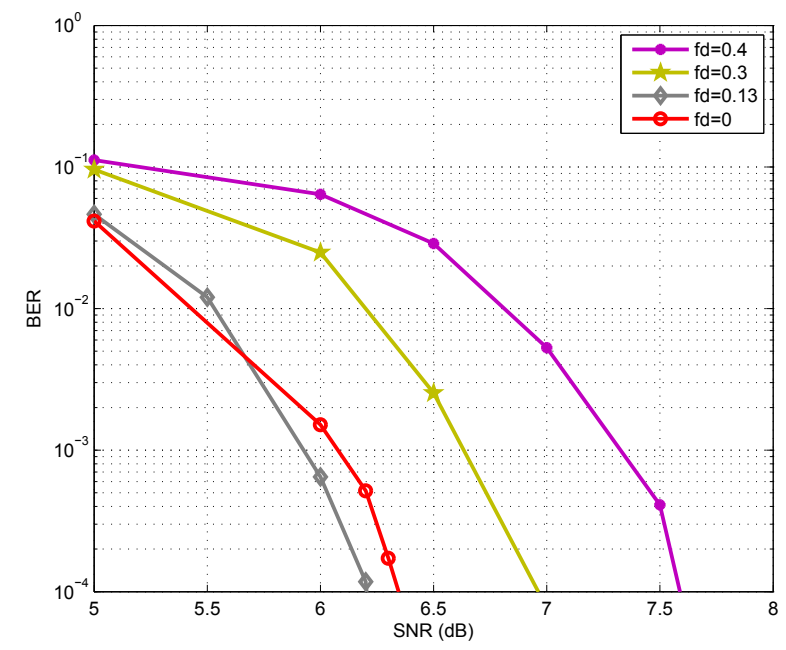

Fig. 3. Performance of the BP detector for three TU6 mobile scenarios. 10 iterations have been performed in the detector.

The performance of the BP detector for TU6 channel and $f_{d}=0.4$ is analyzed in more detail in Fig. 4, which shows how the iterative process of the FG impacts on the BER performance. We can appreciate that beyond the second iteration, the BER curve reaches the water-falling region giving a noticeable performance gain. Over three iterations, the iterative process tends to converge, and there is no noticeable BER gain performing more than 5 iterations. In this figure, it is also depicted the BER curve for the one-tap equalization case (no ICI compensation technique applied) which has an error-floor at $\mathrm{BER}=10^{-1}$.

Simulation results in Fig. 5 show the performance of the loopy FG in terms of BER with respect to the BCJR algorithm. It is shown that the difference is about $0.2 \mathrm{~dB}$ for $f d=0.3$ and less than $0.3 \mathrm{~dB}$ for $f_{d}=0.4$ at BER $=10^{-4}$. Therefore, we conclude that, from a practical point of view, since BP detector curve approaches the BCJR's at high SNR region, there is no need to implement clustering or stretching techniques [10] with the aim of avoiding length 4 cycles.

Finally, we also present simulation results for two mobile RA6 scenarios with $f d=0.3$ and $f d=0.4$. In this case, the BP detector is able to outperform the performance of free-ICI 


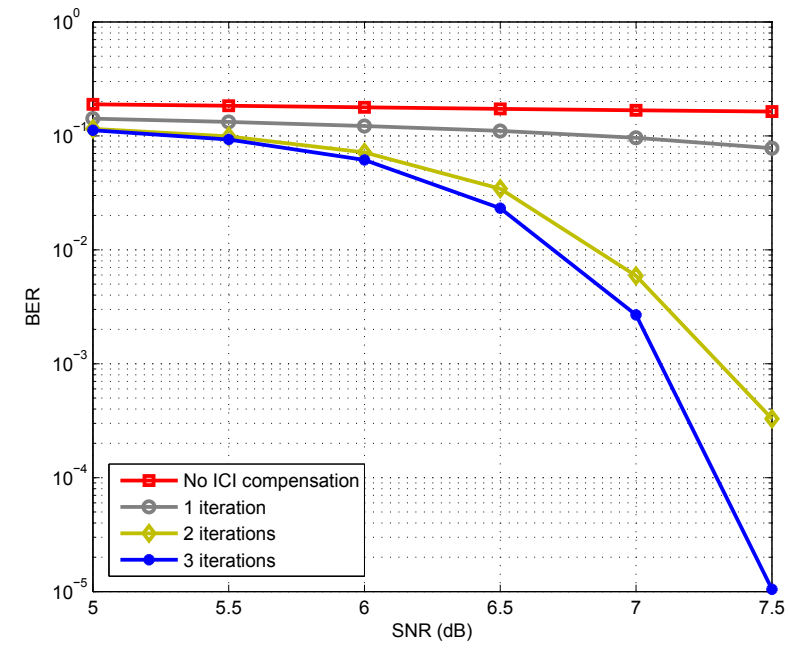

Fig. 4. Performance of the BP detector for different iterations in the FG for TU6 channel.

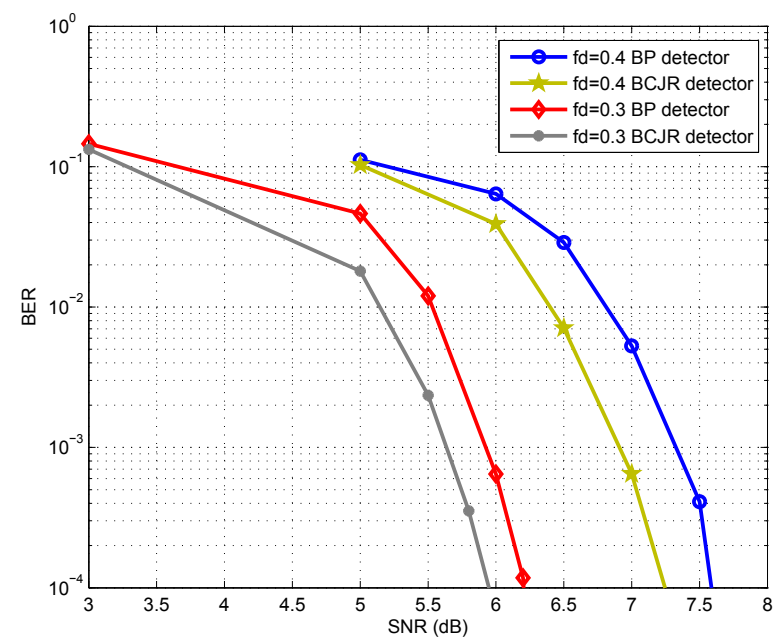

Fig. 5. BER reference of BP detector with respect to optimal BCJR algorithm. TU6 channel is considered.

reception even for the highest Doppler frequency. Therefore, it is concluded that the inclusion of the proposed BP detector in a DVB-T2 receptor is specially worthwhile in rural areas where, besides, the vehicular speed can be higher.

\section{CONCLUSION}

A novel ICI suppressing approach has been proposed for high speed detection at high mobility scenarios, which makes it a good candidate for the mobile reception of DVB-T2 signal, as well as for the future DVB-NGH receptors design. Compared to the traditional BCJR receiver, the proposed FG detector is better suited to large FFT sizes. The performance of the proposed detector has been analyzed over TU6 and RA6 channel models using LDPC codes.

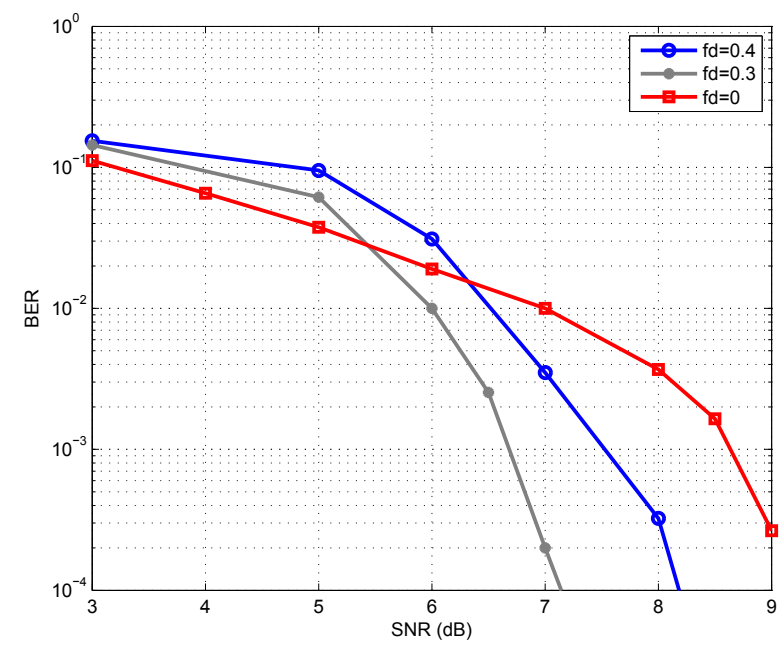

Fig. 6. Performance of the BP detector for two RA6 mobile scenarios.

\section{ACKNOWLEDGMENTS}

The authors want to thank Department of Industry and Innovation and to the Department of Education, Universities and Research of the Basque Government, for its support and funding through IKERTU program.

\section{REFERENCES}

[1] ETSI, "Digital Video Broadcasting (DVB); Frame structure channel coding and modulation for a second generation digital terrestrial television broadcasting system (DVB-T2). ETSI EN 302755 V1.1.1," September 2009.

[2] T. Wang, J. Proakis, E. Masry, and J. Zeidler, "Performance degradation of OFDM systems due to Doppler spreading," IEEE Trans. on Wireless Comm., vol. 5, pp. 1422 - 32, 2006.

[3] D. Liu and M. Fitz, "Iterative MAP equalization and decoding in wireless mobile coded OFDM," IEEE Trans. on Comm., vol. 57, pp. $2042-51,2009$.

[4] S. U. Hwang, J. H. Lee, and J. Seo, "Low complexity iterative ICI cancellation and equalization for OFDM systems over doubly selective channels," IEEE Trans. on Broad., vol. 55, 2009.

[5] K. Fang, L. Rugini, and G. Leus, "Low-complexity block turbo equalization for OFDM systems in time-varying channels," IEEE Trans. on Signal Processing, vol. 56, pp. 5555-66, 2008.

[6] C.-Y. Hsu and W.-R. Wu, "Low-complexity ICI mitigation methods for high-mobility SISO/MIMO-OFDM systems," IEEE Trans. on Vehicular Technology, vol. 58, pp. 2755-68, 2009.

[7] L. Wilhelmsson, J. Svensson, A. Nevalainen, and M. Faulkner, "Some results on implementing low-complex ICI cancellation for DVB-H," IEEE 65th Vehicular Tech. Conf. - VTC2007-Spring, pp. 2931-2935, 2007.

[8] M. Poggioni, L. Rugini, and P. Banelli, "DVB-T/H and T-DMB: physical layer performance comparison in fast mobile channels," IEEE Trans. on Broadcasting, vol. 55, pp. 719-30, 2009.

[9] P. Baracca, S. Tomasin, L. Vangelista, N. Benvenuto, and A. Morello, "Per sub-block equalization of very long ofdm blocks in mobile communications," IEEE Trans. on Comm., vol. 59, pp. 363-8, 2011.

[10] F. Kschischang, B. Frey, and H.-A. Loeliger, "Factor graphs and the sum-product algorithm," IEEE Trans. on Information Theory, vol. 47, pp. 498-519, 2001.

[11] G. Colavolpe and A. Barbieri, "On MAP symbol detection for ISI channels using the Ungerboeck observation model," IEEE Comm. Letters, vol. 9, pp. 720-2, 2005.

[12] M. Kaynak, T. Duman, and E. Kurtas, "Belief propagation over mimo frequency selective fading channels," 2005. 